\title{
CHARACTERISTIC ABSORPTION FEATURES IN THE SPECTRA OF AP-SI STARS BETWEEN 1250 AND $1850 \AA$.
}

\author{
Marie-Christine ARTRU \\ Département d'Astrophysique Fondamentale \\ Observatoire de Meudon \\ F-92195 MEUDON PRINCIPAL CEDEX. France

\begin{abstract}
Thierry LANZ
institut d'Astronomie de I'Université de Lausanne and Observatoire de Genéve.
\end{abstract} \\ $\mathrm{CH} 1290$ CHAVANNES-DES-BOIS. Switzerland
}

\begin{abstract}
On the basis of IUE data. the specific absorption features in the spectra of Ap-Si Stars are displayed in the 1250-1850 $\AA$ range and identified when possible. The contribution of the Si II multiplets is calculated in LTE for a typical overabundance $\times 10^{2}$ of silicon. It accounts for only a small part of the observed absorption. Commun unidentified structures. smaller than the $1400 \AA$ depression are pointed out.
\end{abstract}

\section{INTRODUCTION}

The broad absorption features observed at $1400 \AA$ in the spectra of magnetic Ap stars is well correlated to the silicon overabundance (Jamar et al. 1978). It is interpretated as autoionization of Si II (Artru et al. 1981) but the precise identification cannot be established without new laboratory data on the highly excited levels of $\mathrm{Si}^{+}$. Our purpose is to derive from IUE spectra a refined description of all the absorption features that appears specific of Ap-Si stars in the spectral range 1250-1850 $\AA$.

More than 150 different stars. classified as Ap or Bp. have been observed by the IUE satellite. From these archives. we have selected a set of sillicon stars with effective temperatures in the $10000-14000 \mathrm{~K}$ range and large absorption features at $1400 \AA$. We present here preliminary results obtained for the limited data sample described in table 1.

\section{DESCRIPTION OF THE AP-SI SPECIFIC ABSORPTION}

To exhibit the specific absorptions in the silicon stars atmospheres. we display "relative" spectra by plotting the quantity $\log \left(F_{\lambda}(p) / F_{\lambda}(n)\right)$ versus the wavelength $\lambda: F_{\lambda}(p)$ is the observed spectral flux of the peculiar star and $F_{\lambda}(n)$ the same for a normal comparison star of similar effcctive temperature. We use calibrated net spectra. either directly obtained from the IUE low-resolution images. Or derived from highresolution images after degrading the resolution.

Such "relative" spectra are given in fig. 1. The curves a, b and c correspond to the three silicon stars of table 1 and show the striking similarity of their absorption features. For comparison the curve od relates to each other the two normal stars (HD 87901 and HD 17081).

\section{5}

C. R. Cowley et al (eds.), Upper Main Sequence Stars with Anomalous Abundances, 105-108.

(C) 1986 by D. Reidel Publishing Company. 


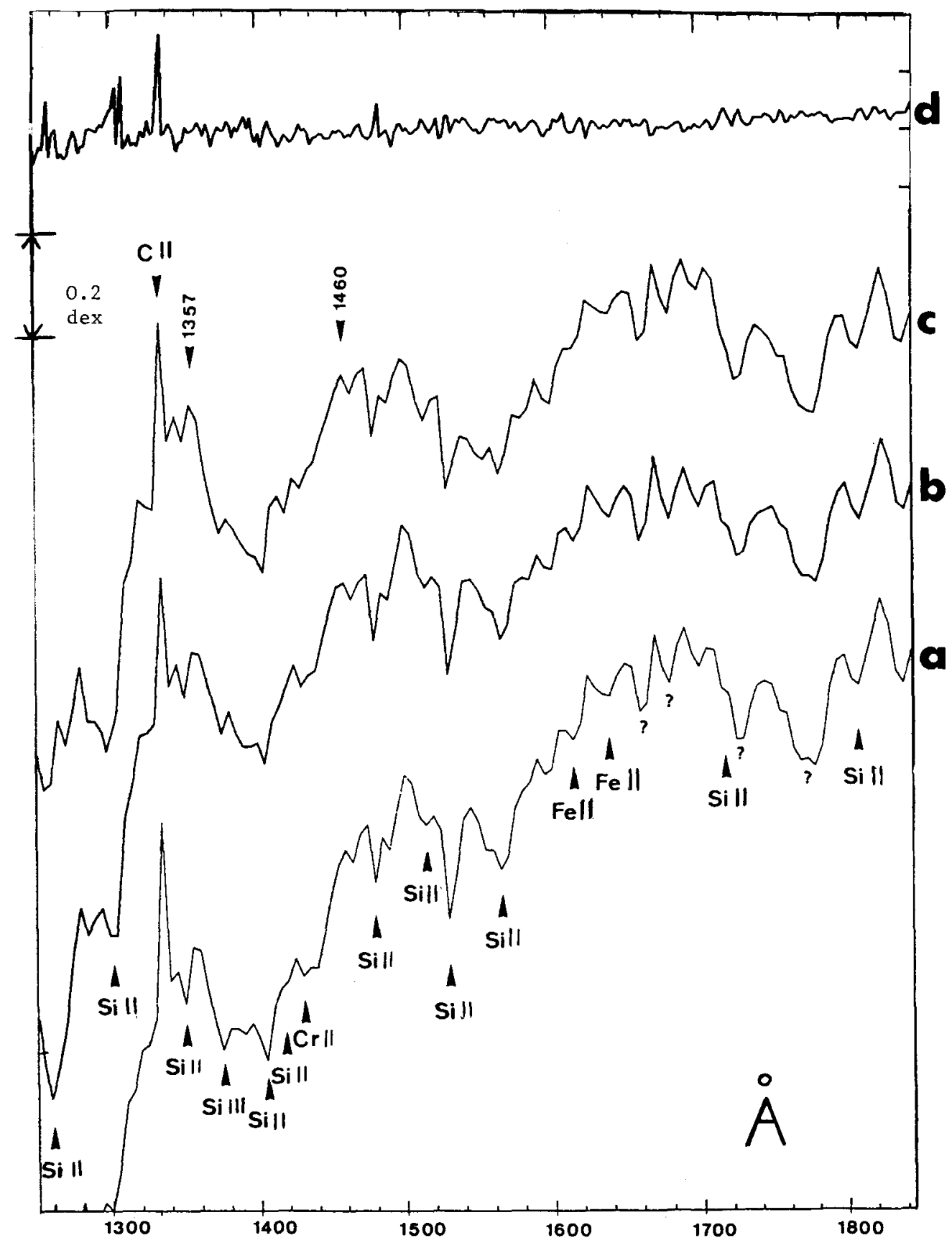

Fig. 1 - Relative spectra of three Ap-Si stars (a, b, c) and one normal star (d). The logarithm of the flux ratio is plotted for HD27309/HD17081 (a), HD25267 / HD17081 (b), HD34452/HD17081 (c) and HD87901/HD17081 (d). 


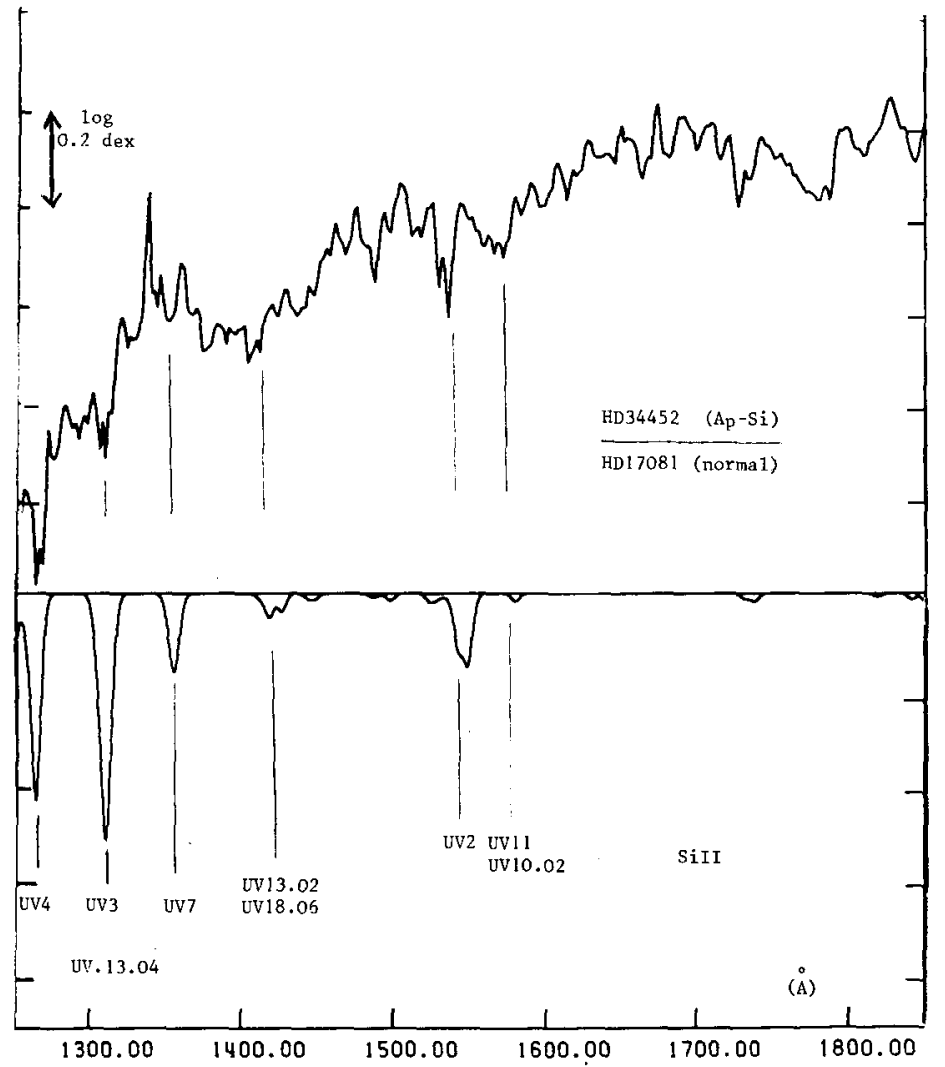

Fig. 2 - LTE synthetic spectrum of $\mathrm{Si}$ II multiplets for a silicon abundance of
$100 \times$ (lower curve) and observed "relative" spectrum of HD34452 (upper curve) (the resolution is about $6 \mathrm{~A}$ ).

Table 1 - Sample of Ap-Si stars and IUE observations

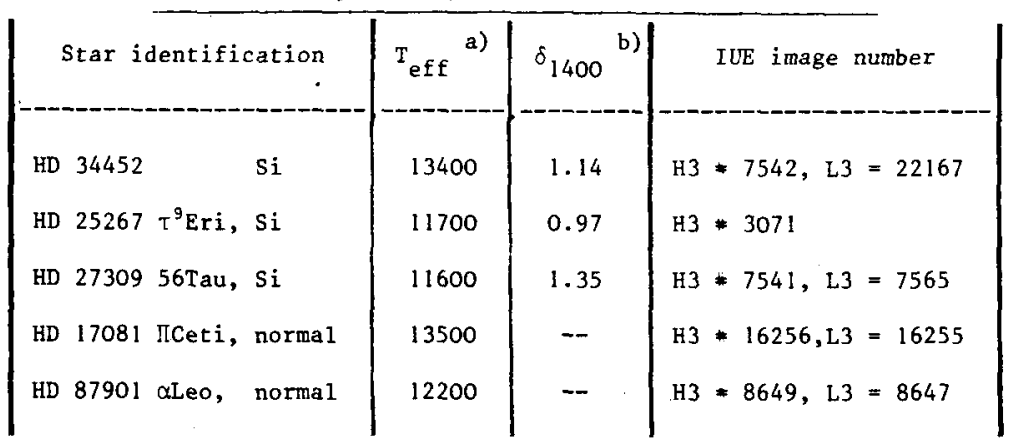

a) The effective temperature is estimated fromphotometric indexes (Lanz, 1985).

b) The $\delta_{1400}$ index measures the enhancement of the $1400 \AA$ depression (Jamar et al, 1978). 
Many of the sharper absorption lines, in the "relative" Ap -Si star spectra (fig. 1-a, b and c) are identified as strong multiplets of overabundant ions. mainly Si II and Fe II. The C II multiplet at $1335 \AA$ appears as a peak. suggesting an underabundance of carbon. A similar weaker feature also appear for the comparison star HD 87901 (flg. 1-d).

The large $1400 \AA$ feature has a similar shape for the three sillcon stars and occurs between the same limits. 1357 and $1460 \AA$. Other smaller wide depressions are observed similarly for the three SI stars. around $1550 \AA$ and $1750 \AA$. Their identification. possibly by autolonization of an overabundant ion is still an open question.

\section{THE ABSORPTION SPECTRUM OF SI $\|$}

The strongest lines identified on the observed curves of fig. 1 are due to Si II multiplets (Moore. 1965) : for instance. UV4 at $1264 \AA$. UV 13.02 and UV13.03 at $1405-1410 \AA$. UV12 and UV15. O4 at $1485 \AA$ and UV2 at $1530 \AA$.

in order to evaluate the contribution of the Si II spectrum to the opacity variations observed in fig. 1. we first calculated the total effect of the "normal" lines. as known from laboratory analysis and complled by Moore (1965). A predicted LTE synthetic spectrum of SI II was computed using a blanketed model $(13000 \mathrm{~K}, \log g=4)$ from Kurucz et al (1977). The silliclum abundance was fixed at 100 times the solar one. Which is the value generally given for HD 34452. The line list was limited to the experimental lines of SI II. with the gf-values taken from the compliation of Lanz and Artru (1985). The line spectrum of Si II obtained in this calculation is shown on fig. 2. with the same resolution and scale. as for the observed curves of fig. 1. Their comparison confirms the identification of the Si II multiplets, but shows that they contribute for only a small part. to the observed opacity. The "normal" spectra of other overabundant ions should also be calculated. but are likely not to fill the missing opacity. A systematic study of their autoionization spectra would be essential.

\section{REFERENCES}

- Artru. M. -C. Jamar, C. . Petrini. D. . Praderie. F. . 1981. Astron. Astrophys. 96. 380 .

- Jamar. C. Macau-Hercot. D. Praderie. F., 1978. Astron. Astrophys. 63. 155.

- Kurucz. R. L. . Peytremann. E. . Avrett. E. H. . 1974. Blanketed Model Atmospheres for Early-type Stars (Washington. Smithsonian Institution Press).

- Lanz. T. . 1985. Astron. Astrophys. 144. 191.

- Lanz. T. Artru. M. - C. . 1985. Physica Scripta. in press.

- Moore. C. E. . 1965. Selected Tables of Atomic Spectra. NSRDS-NBS3. National Bureau of Standards. 